\title{
Potential Diagnostic and Prognostic Biomarkers of Circular RNAs for Lung Cancer in China
}

\author{
Chengdi Wang $\mathbb{D}^{1},{ }^{1}$ Yuting Jiang, ${ }^{2}$ Qian Lei, ${ }^{3}$ Yangping Wu, ${ }^{4}$ Jun Shao, \\ Dan Pu $(1),{ }^{5}$ and Weimin $\mathrm{Li}\left(\mathbb{C}^{1}\right.$ \\ ${ }^{1}$ Department of Respiratory and Critical Care Medicine, West China Medical School/West China Hospital, Sichuan University, \\ Chengdu, China \\ ${ }^{2}$ West China Medical School, Sichuan University, Chengdu, China \\ ${ }^{3}$ Department of Targeted Tracer Research and Development Laboratory, West China Hospital, Sichuan University, Chengdu, China \\ ${ }^{4}$ Department of Clinical Research Management, West China Hospital, Sichuan University, Chengdu, China \\ ${ }^{5}$ Clinic Skill Center, West China Hospital, Sichuan University, Chengdu, China
}

Correspondence should be addressed to Dan Pu; pudanHUAXI@yeah.net and Weimin Li; weimi003@yahoo.com

Received 15 February 2019; Revised 20 May 2019; Accepted 10 June 2019; Published 25 August 2019

Academic Editor: Yujiang Fang

Copyright (C) 2019 Chengdi Wang et al. This is an open access article distributed under the Creative Commons Attribution License, which permits unrestricted use, distribution, and reproduction in any medium, provided the original work is properly cited.

Emerging evidence demonstrated that circular RNAs (circRNAs) were dysregulated in lung cancer, indicating that circRNAs might serve as novel diagnostic and prognostic biomarkers for lung cancer. However, the clinical value of circRNAs on lung cancer remains unclear. This study aimed to evaluate the efficiency of circRNAs in the diagnosis and prognosis for lung cancer in China. 2122 Chinese individuals were enrolled in this investigation for assessment of diagnostic value and examination of prognostic analysis. In the diagnostic analysis, the pooled sensitivity, specificity, PLR, NLR, DOR, and AUC of the sROC curve with their 95\% CIs were 0.80 (95\%CI: 0.74-0.84), 0.80 (95\%CI: 0.73-0.86), 3.97 (95\%CI: 2.80-5.62) and 0.26 (95\%CI: 0.19-0.34), 15.51 (95\%CI: 8.76-24.47), and 0.85 (95\%CI: 0.82-0.88), respectively. As for the prognostic power of circRNAs, lung cancer patients with higher expression levels of circRNAs tend to possess lower overall survival with the overall pooled HR (1.70, 95\%CI: 1.26-2.29). Furthermore, in stratified analysis, upregulated and downregulated circRNAs were manifested to exert significant effects on prognosis with HR values of 2.17 (95\%CI: 1.74-2.72) and 0.52 (95\%CI: 0.34-0.80). This study validates that circRNAs are promising diagnostic and predictive biomarkers for lung cancer patients in China.

\section{Introduction}

Lung cancer is the most common of new cancer cases accounting for $11.6 \%$ of the new diagnosed cases and ranks as the leading cause of cancer death sharing $18.4 \%$ of the overall cancer mortality [1]. By sex, in males, lung cancer is the top one cancer type, responsible for newly diagnosed cancer patients and death, whereas lung cancer is the second death cause inferior to breast cancer and as for incidence rate comes in third behind breast cancer and colorectum cancer among females [1]. Obviously, lung cancer has been a major public health problem worldwide especially in China [2].

Despite the considerable efforts exerted on dealing with cancer, there are still clinical challenges in cancer management, mainly ascribed to low early metaphase diagnosis rate with cancer, ineffective treatment, and uncertainty about clinical outcomes. Early diagnosis can make a huge difference to lung cancer patients, for providing the best opportunity for medical support [3]. If diagnosed at early stage, lung cancer patients with mild symptoms may be protected from developing severe, late-stage, and advanced cancer types, which will tend to require more intricate and expensive treatment with poorer curative effects. Owing to the reasons mentioned above, traditional treatments are not satisfactory [4]. Immunotherapy, stem cells, and genomic medicine are emerging as novel attractive candidate strategies against cancer with striking treatment effect. But more importantly, there are yet a substantial number of obstacles to overcome, such as second developed drug resistance, prior to entering the clinic and being widely employed. Thus, effective methods 
or biomarkers are in extremely urgent need for early diagnosis and prognosis of lung cancer, so as to monitor the progress of cancer and adjust treatment plan timely.

Circular RNAs (circRNAs) are emerging as a promising biomarker for cancers [5]. CircRNAs distinctly feature covalently closed continuous loop structures without 3' ends and 5' ends, while, in well-established linear RNAs, another important member of the family of endogenous noncoding RNAs, 3' ends and 5' ends exist, limit the direction of synthesis of nucleic acids in vivo to 5'-to-3', and contribute to linear RNAs' sensitivity to nuclease [6,7]. At first, circRNAs were noted as abnormal byproducts of back-splicing of premRNA transcription because pretty low expression levels of circRNAs were observed [8]. However, with the burgeoning development and incremental application of novel technologies, especially the high-throughput RNA sequencing, altered circRNAs are confirmed to be ubiquitously expressed $[9,10]$.

More importantly, the distinct molecular structure grants circRNAs multiple nature, including stability, specificity, and conservation across mammals $[9,11]$. Compared with linear RNAs, circRNAs can avoid exonucleolytic degradation by RNase; thereby, they tend to possess longer half-time and then are able to stay more stable in vivo over an extended period [12], which partly conduce to plentiful expression in internal environment. Moreover, circRNAs are deemed to be dispersed in a cell/tissue-dependent manner and their expression levels vary, which is consistent with specific developmental stages [12-14], which are related to their extensive biological functions, involved in cell proliferation, differentiation, migration, invasion, and apoptosis [15-21]. At the same time, increasing evidence, focused on the correlation between circRNAs and clinical characteristics of cancer sufferers, revealed that circRNAs might act as effective diagnostic biomarkers and forecast clinical outcomes of cancer patients [22]. Zhao et al. [23] screened 357 differentially expressed circRNAs by high-throughput sequencing in early lung adenocarcinoma. They further investigated 5 circRNAs by bioinformatic analysis and reported that these circRNAs might function as diagnostic markers in cancer. What is more, circFOXO3, a putative tumor suppressor, was significantly downregulated in lung cancer and breast cancer $[24,25]$. Zhang et al. reported that circFOXO3 served as a novel biomarker for early diagnosis with AUC of 0.782 in lung cancer and in vitro investigations implied inversely correlation with migration and invasion of nonsmall cell lung cancer through sponging miR-155 and releasing FOXO3 gene [24]. Besides, CiRS-7 (circular RNA sponge for miR-7), also termed CDRlas (cerebellar degeneration-related protein 1 transcript), harbors more than 70 conventional miR-7 binding sites and directly suppresses activity of miR-7 [26]. Apart from being a well-known tumor suppressor, miR-7 is also reported to show the opposite influence effect in lung cancer [26-28], colorectal cancer [29, 30], and hepatocellular carcinoma [31]. Upregulated expression levels of CDRlas with concomitant underexpressed mir-7 were proved to closely relate to high TNM stage, lymph nodes metastasis, and short survival time [32]. On the contrary, Chou and his colleagues identified overexpression of miR-7 with carcinogenesis and poor prognosis of lung cancer. Analogously, in the context of inhibition of miR-7, there was reduced proliferation of lung cancer cell lines [28].

Thus, there are disagreements and inconformity among the results of diverse studies concentrated on the diagnostic ability and prognostic value of circRNAs. Here, we performed a comprehensive and quantitative study to summarize the diagnostic and prognostic utility of circRNAs in human lung cancer specifically, tried to clarify and address the discrepancy among researches, and expected to furnish guideline to clinical management of lung cancer.

\section{Materials and Methods}

2.1. Search Strategy and Study Selection. A comprehensive search was conducted to identify potential articles published in English up to December, 2018, from PubMed, PMC, EMBASE, Web of Science, Cochrane Library, China National Knowledge Infrastructure Database (CNKI), Wanfang Database, and China Biological Medicine Database (CBM). The search terms employed for literature retrieval were (circRNA OR circular RNA) AND (lung cancer OR lung carcinoma OR pulmonary carcinoma OR pulmonary cancer OR lung squamous cell carcinoma OR non-small-cell lung cancer OR small cell lung cancer). Reference lists of relevant papers were obtained manually to identify potential eligibility.

Two investigators (Y. T. Jiang and J. Shao) independently perused the full texts of potentially eligible studies based on their titles and abstracts. Any disagreement was resolved until a consensus was reached with a third researcher (C. D. Wang).

Publications included in this meta-analysis fulfilled the following criteria: (1) case-control study or cohort study including both case and control groups; (2) patients with a pathological diagnosis of lung cancer; (3) studies estimating performance of circRNAs for the diagnosis or predicting the outcome of lung cancer patients; (4) the sensitivity and specificity data or HRs with 95\% CI (or the possibility of deriving such statistics from the manuscript) that were available. And exclusion criteria included (1) studies not relevant to circRNA or lung cancer; (2) key information or usable data that were missing; (3) duplicated publications; (4) reviews, letters, case reports, summaries of conference, and so on. If articles were published based on overlapping data by the same author, only the most complete study was included.

2.2. Data Extraction and Quality Assessment. Data are collected according to different study types.

(I) In studies using circRNAs as diagnostic marker for lung cancer, following data were enrolled: the first author, publication year, country and ethnicity, cancer type, specimen source, sample size, cut-off value, area under the curve (AUC), data for $2 \times 2$ contingency table (sensitivity and specificity), and detection method.

(II) In these articles assessing prognostic significance of circRNAs in lung cancer, we extracted following information: the first author, publication year, country and ethnicity, cancer type, specimen source, sample size, cut-off value, followup time (month), treatment, and HR values of evaluated 


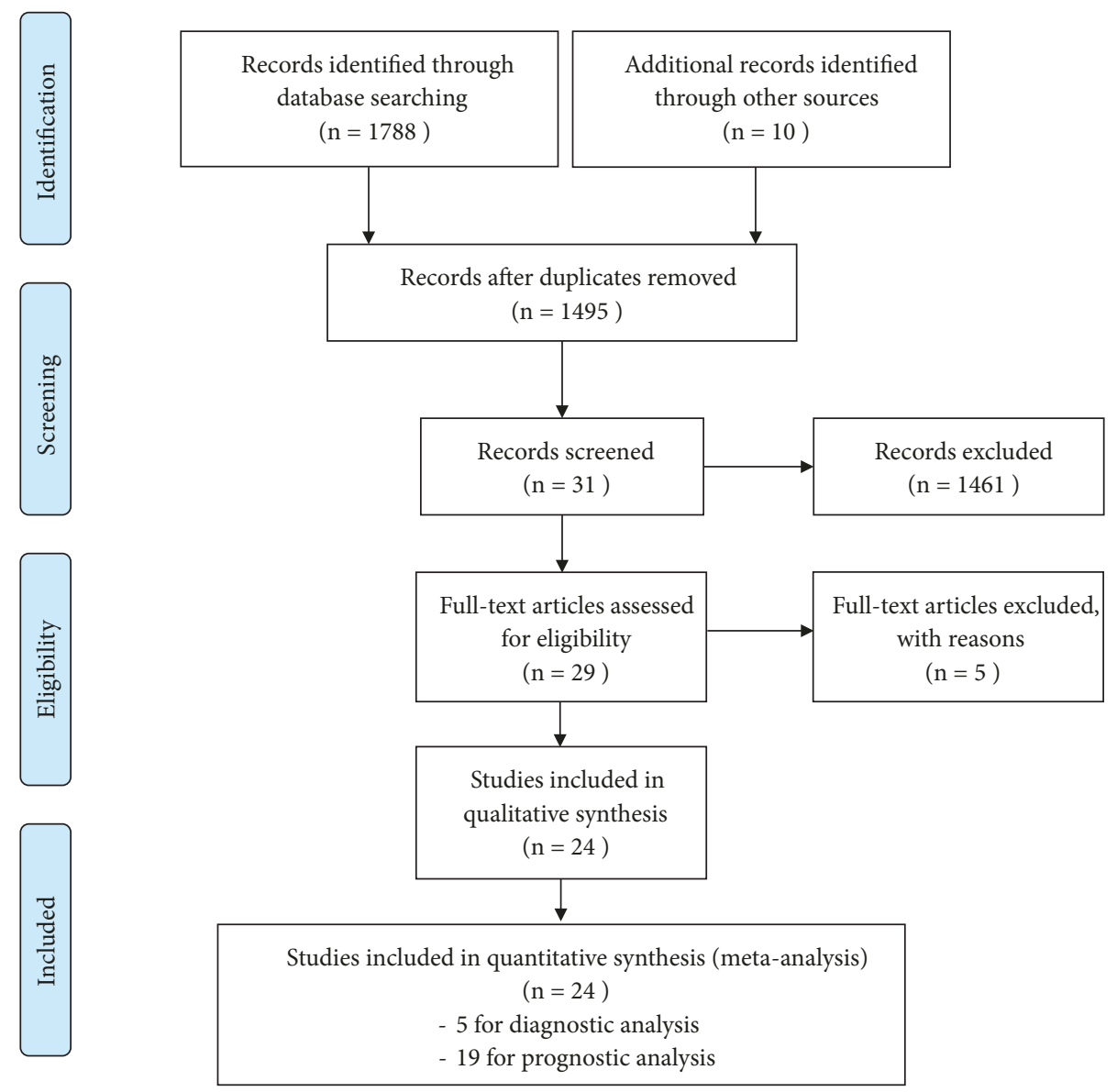

FIgURE 1: Flow diagram of the study selection process. Totally, 1798 were identified through database or by searching manually. And 24 articles were enrolled in the final analysis including 5 diagnostic studies and 19 prognostic studies.

circRNAs for overall survival (OS) analysis as well as their 95\% CI and P value.

Two researchers reviewed and evaluated the quality of studies enrolled in prognostic analysis based on the guideline of The Quality Assessment of Diagnostic Accuracy Studies 2 (QUADAS-2) checklist. QUADAS-2 consists of 14 items, and there is an answer of "Yes," "unclear," and "No" for each item for which only "Yes" scores one point. The NewcastleOttawa Quality Assessment Scale (NOS) was adopted to systematically assess articles included in the prognostic metaanalysis. Specifically, the cut-off point is defined as 6. Higher scores represent better reporting quality.

2.3. Statistical Analysis. All statistical analyses were performed with STATA version 15.0 (STATA Corporation LLC, Texas, USA) and Review Manager 5.3 (Cochrane Collaboration, London, UK). Pooled sensitivity, specificity, positive likelihood ratio (PLR), negative likelihood ratio (NLR), diagnostic odds ratios (DOR) and their $95 \%$ confidence interval (CI), summary receiver operator characteristic (SROC) curve, and area under the curve (AUC) were calculated to estimate the ability of circRNA to distinguish lung cancer patients from healthy people. As for survival rates, all provided HRs as well as $95 \%$ CI were obtained to study the overall performance of the prognostic test. $\mathrm{P}<0.05$ (two-sided) was considered as a statistically significant difference. Heterogeneity across studios was tested by Cochran's Q test and Higgins's $I^{2}$ statistics. A random-effect model was utilized when $\mathrm{P}<0.10$ and $I^{2}>50 \%$, indicating the presence of heterogeneity. Otherwise, the fixed-effect model was carried out. Finally, publication bias was described by Egger's bias indicator test.

\section{Result}

3.1. Study Selection. A total of 1798 potentially relevant articles were initially identified. After abstract and full article review, 24 published articles were enrolled for the final analysis. Among them, 5 articles investigated diagnostic value of circRNAs in lung cancer [24, 33-36], and 19 studies examined prognostic information related to overall survival [32, 37-54]. The period of the eligible studies ranged from 2017 to 2018 with a total of 2122 individuals. The process of selection is shown (Figure 1).

3.2. Study Characteristics and Quality Assessment. The characteristics of 24 eligible studies are summarized in Tables 1 and 2. Among them, 20 circRNAs were upregulated 


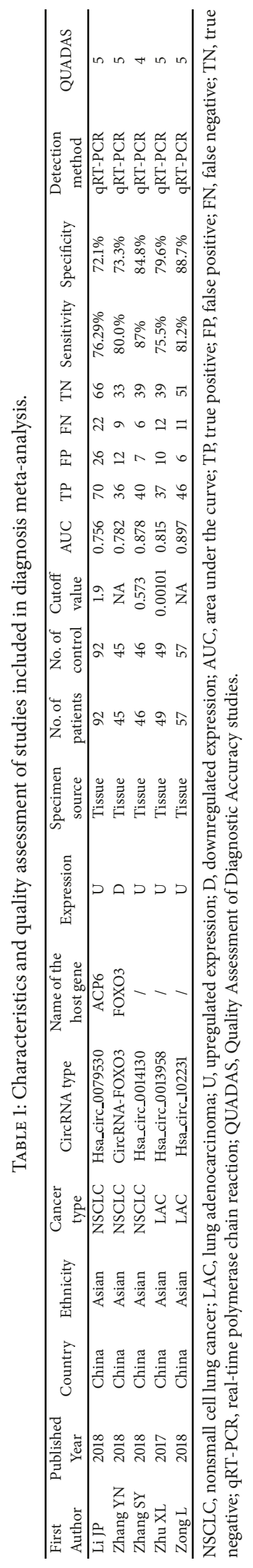




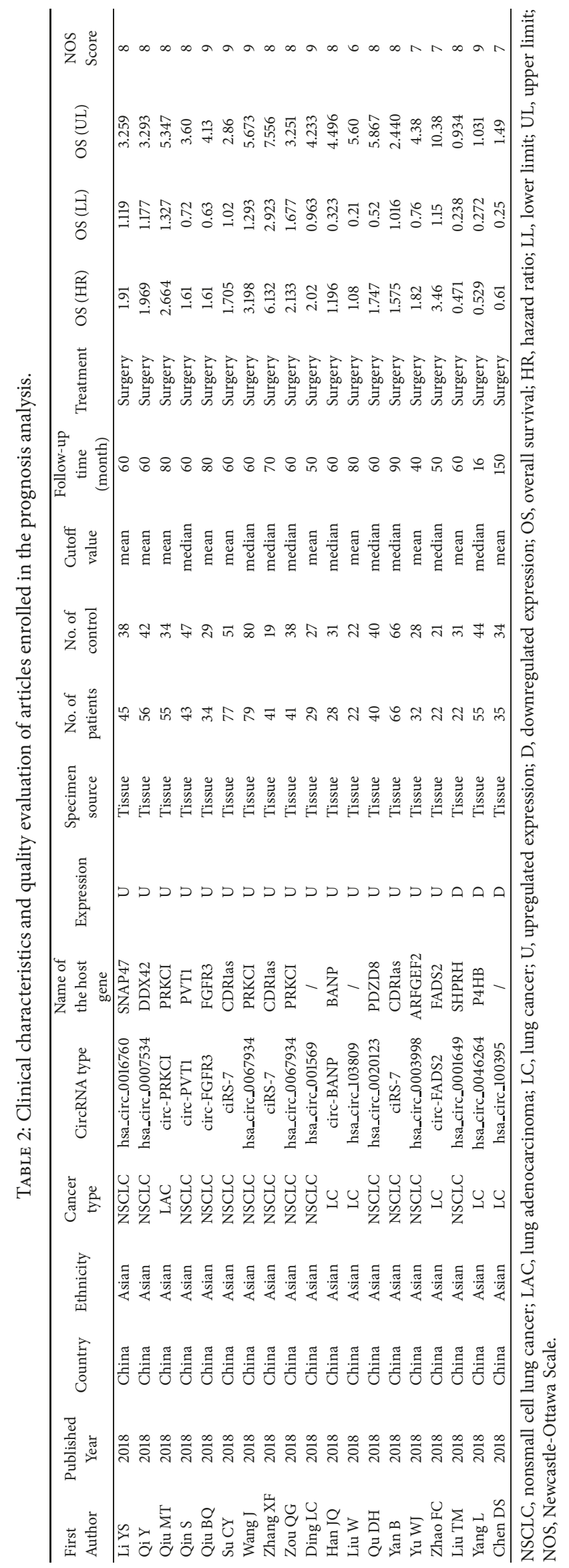




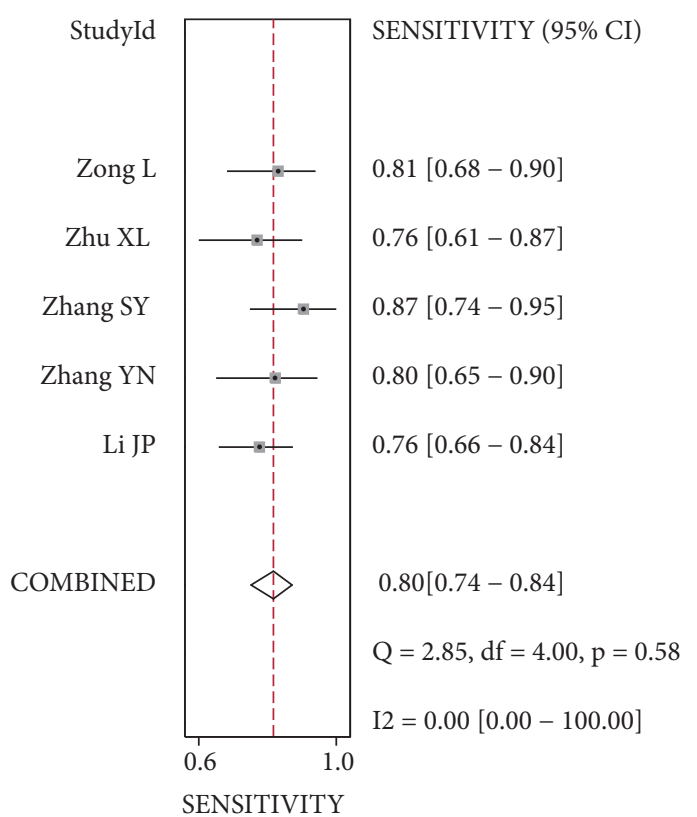

(a)

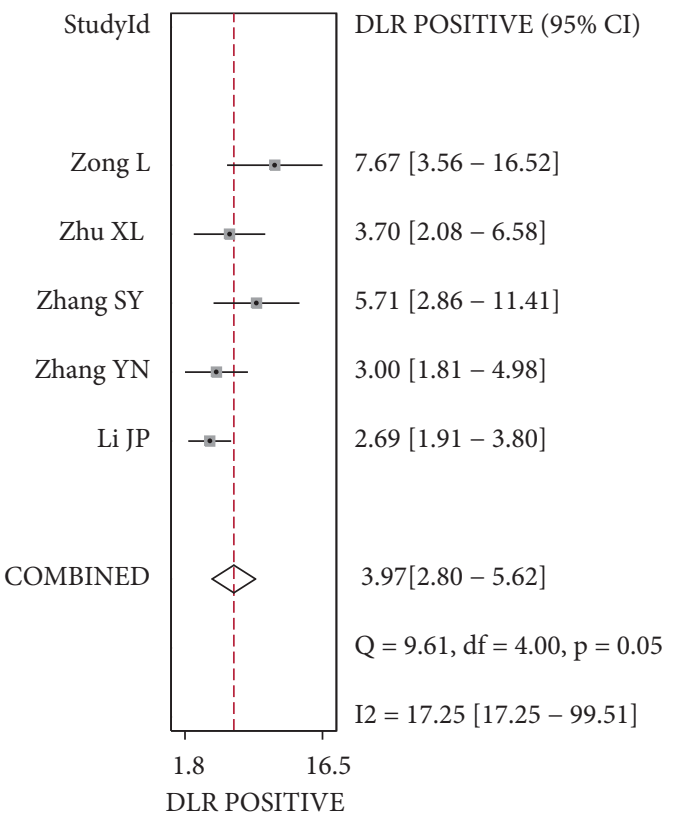

(c)

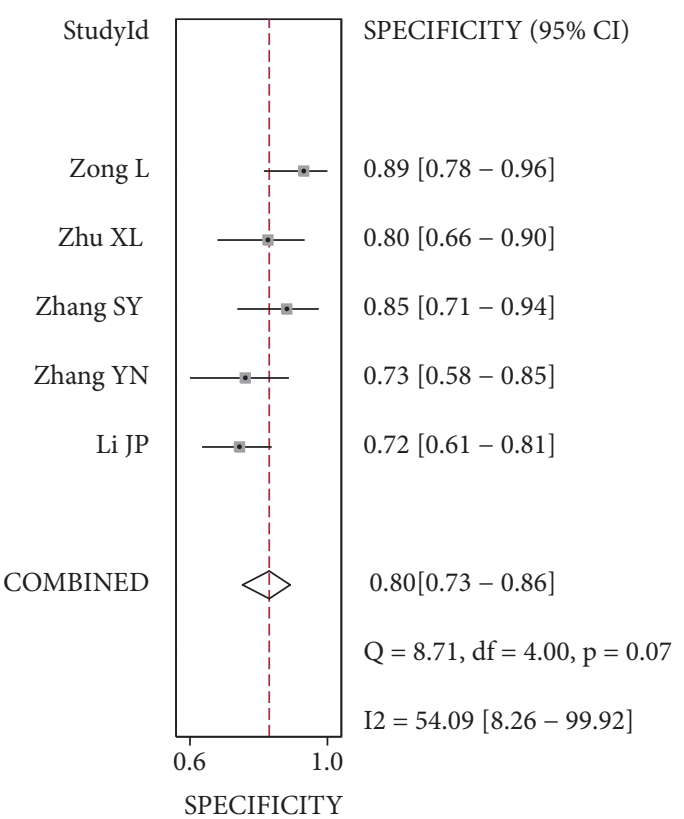

(b)

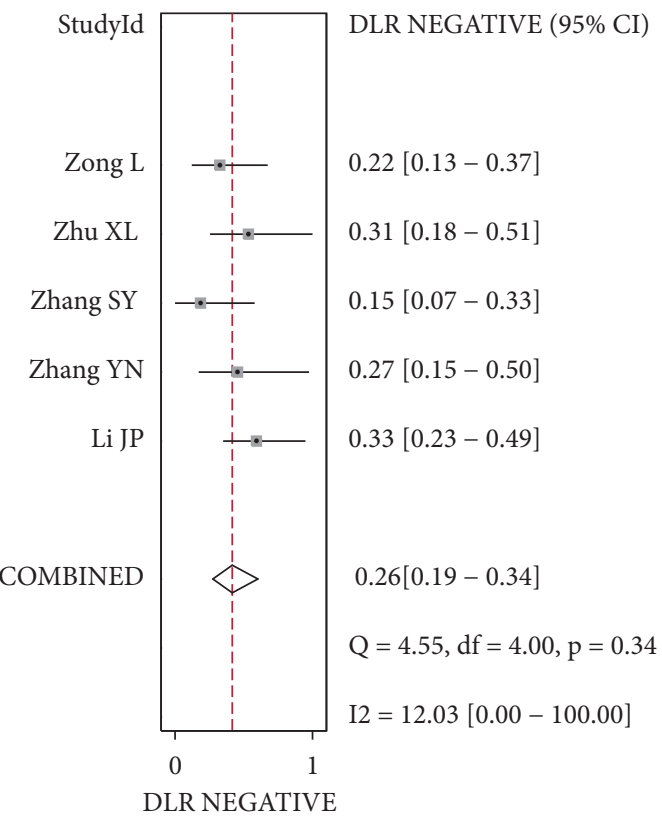

(d)

FiguRE 2: Forest plots of diagnostic accuracy index of circRNAs in lung cancer. (a) Sensitivity of circRNAs in diagnosis of lung cancer. (b) Specificity of circRNAs in diagnosis of lung cancer. (c) Positive likelihood ratio of circRNAs in diagnosis of lung cancer. (d) Negative likelihood ratio of circRNAs in diagnosis of lung cancer.

in lung cancer patients while 4 types were downregulated (circFOXO3, Hsa_circ_0001649, Hsa_circ_0046264, and Hsa_circ_100395). All of the sources of sample were tissue. The sample size ranged from 43 to 184 . And the overall size in diagnostic meta-analysis was 578 and in the patients involved in prognostic analysis it was 1544 . The cut-off values were not consistent in included studies. Additionally, we evaluated the quality of publications concerning diagnosis by QUADAS-2 (Figure S1) and detailed information was shown in Table 1, demonstrating reliable foundation of this study. The quality of prognostic articles was assessed by NOS, and quality scores more than 6 were recognized as high quality in Table 2 . The median of involved studies was 8 , which indicated that the inclusive articles were of good quality.

3.3. Diagnostic Accuracy Analysis. The pooled sensitivity (Figure 2(a)), specificity (Figure 2(b)), PLR (Figure 2(c)), and 


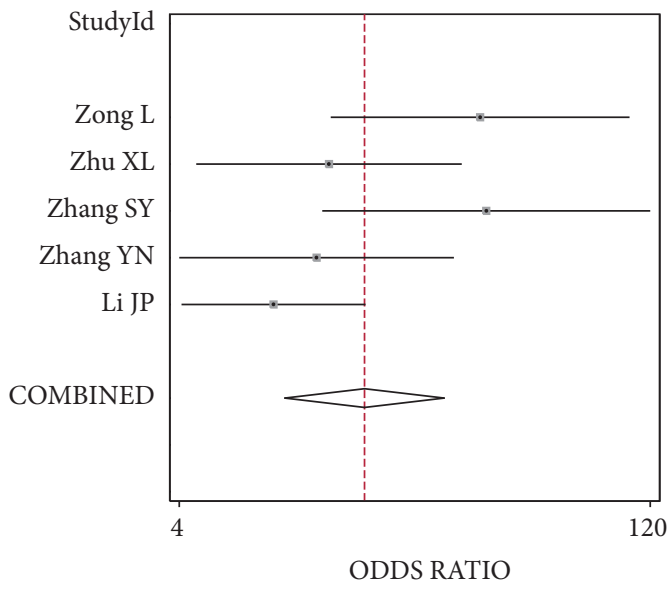

ODDS RATIO (95\% CI)

35.55 [12.17 - 103.79]

12.03 [4.64 - 31.16]

$37.14[11.46-120.42]$

$11.00[4.11-29.45]$

8.08 [4.18 - 15.63]

$15.51[8.76-27.47]$

$\mathrm{Q}=59.95, \mathrm{df}=4.00, \mathrm{p}=0.00$

I2 $=93.33[89.07-97.59]$

(a)

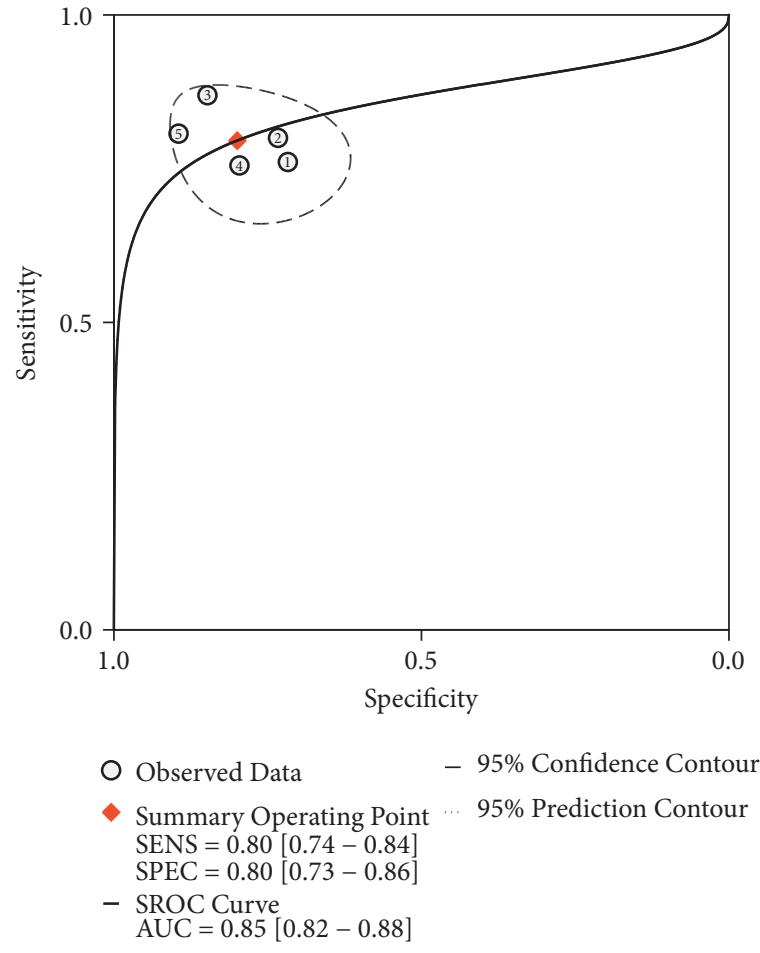

(b)

FIGURE 3: Overall performance of circRNAs in diagnosis of lung cancer. (a) Diagnostic odds ratio of circRNAs in diagnosis of lung cancer. (b) Summary receiver operator characteristic curve of circRNAs in diagnosis of lung cancer.

NLR (Figure 2(d) ) with their 95\% CIs were 0.80 (95\%: 0.740.84 ), 0.80 (95\%: 0.73-0.86), 3.97 (95\%: 2.80-5.62), and 0.26 (0.19-0.34), respectively. The pooled DOR (Figure 3(a), 15.51, 95\%CI: 8.76-27.47) and AUC (Figure 3(b), 0.85, 95\%CI: 0.820.88 ) of the SROC curve were utilized to assess the overall diagnostic performance. Nomogram of Fagan was utilized and the results were demonstrated (Figure 4). The diagnostic performance was summarized in Table 3.

3.4. Prognostic Value of CircRNA Expression for Cancer Survival. Totally, 10 studies provided reported overall survival data and 9 articles concerning Kaplan-Meier curves were calculated to obtain HRs and their 95\%CIs. The pooled $\mathrm{HR}$ is 1.70 (95\%:1.26-2.29) with significant heterogeneity $\left(I^{2}: 72.8 \%\right)$. The overall performance of circRNA as a prognostic biomarker was illustrated (Figure 5). Thus, subgroup analysis was conducted in Table 4. First, upregulated and downregulated circRNAs were analyzed to obtain their HRs values (Figure 6(a)). The recalculated HRs are 2.17 (95\%CI: 1.74-2.72) and 0.52 (95\%CI: 0.34-0.80) with low heterogeneity $\left(I^{2}: 43.2 \%\right.$ and $0.00 \%$, respectively). And there was obviously statistical significance either in multivariate analysis or in univariate analysis (p: 0.007, Figure 6(b)). There was a significant association between more than 5-year period 


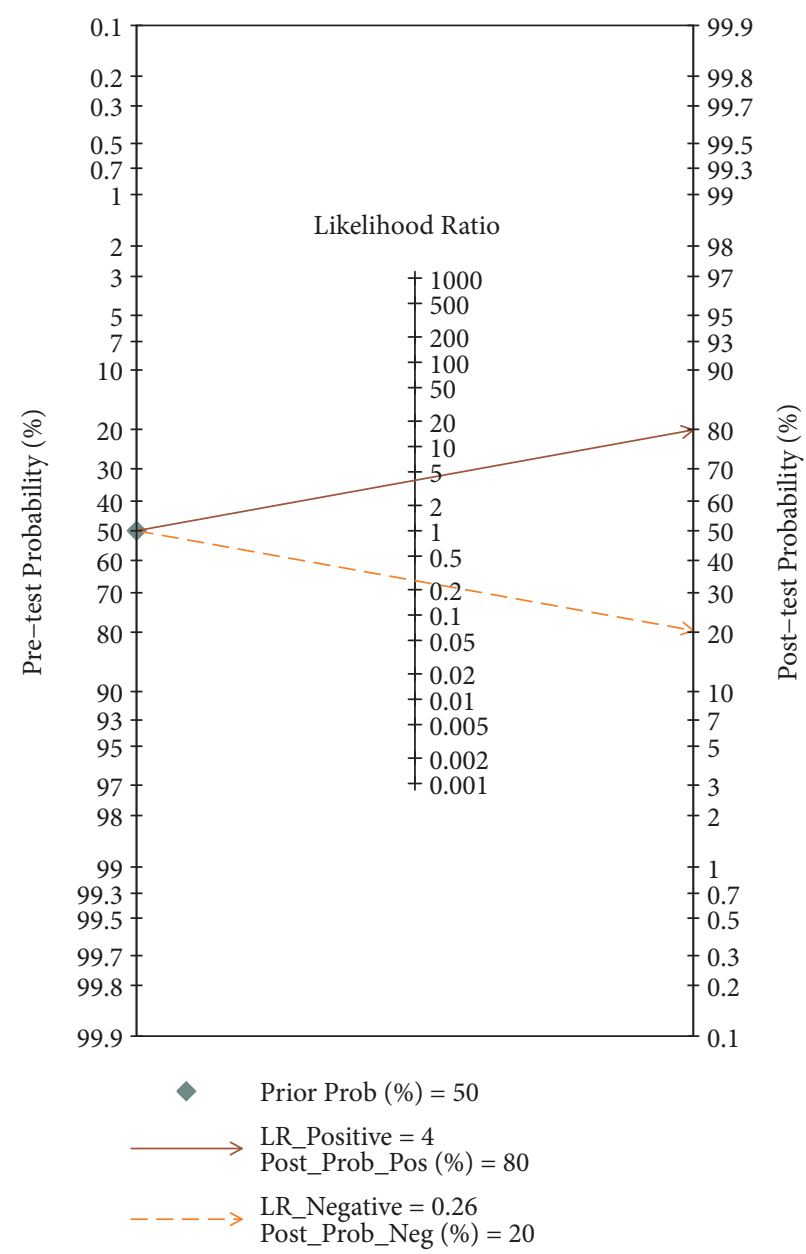

FIGURE 4: Fagan's nomogram estimating the overall value of circRNAs in cancer detection.

time and survival, indicating 5-year follow-up is necessary (Figure 6(c)).

3.5. Publication Bias and Sensitivity Analyses. The publication bias of diagnostic studies was checked by Deeks' test $(\mathrm{P}=0.34$, Figure $7(\mathrm{a})$ ), indicating no potential bias. As for prognostic articles, the $\mathrm{p}$ values of Begg's and Egger's test were 0.484 and 0.339 , respectively (Figures $7(\mathrm{~b})$ and $7(\mathrm{c})$ ). Thus, there were no publication biases in the studies enrolled in the current study. Then, through successively omitting each prognostic individual study, the consequence was not significantly influenced, indicating that the result of this study was robust (Figure 8).

\section{Discussion}

As a member of noncoding cancer genomes, circRNAs gradually attract worldwide attention because accumulating evidence revealed various functions of circRNAs with an emphasis on their association with cancer. Due to being insensitive to RNase, circRNAs tend to keep stable and specifically exist in the plasma of lung cancer patients like F-circEA, which implies that circRNAs may be employed as noninvasive diagnostic biomarkers [12, 55]. Ubiquitously existing in body, altered expression levels of circRNAs are disease specific or often predict prognosis [56]. Therefore, circRNAs may be used as biomarkers so as to facilitate early diagnosis and improvement on prognosis of lung carcinoma. Previous reviews focused on correlation between circRNAs and multiple cancers, but none of them investigated on lung cancer specifically. We summarized recent studies of circRNAs in lung cancer, highlighting circRNAs as diagnostic and prognostic tools. Thus, this study is the first metaanalysis to direct at and summarize the potential diagnostic and prognostic roles of circRNAs for human lung cancer specifically, hoping to contribute to a better and deeper understanding of the complex relationship between the various expression levels of circRNAs and lung cancer.

4.1. CircRNAs Are Diagnostic Biomarkers for Lung Cancer. We retrieved 5 published articles pertaining to the expression levels of different circRNAs in human lung cancer, including 1 downregulated circRNA [24] and 4 upregulated circRNAs [33-36]. Furthermore, in the selection process for eligible 


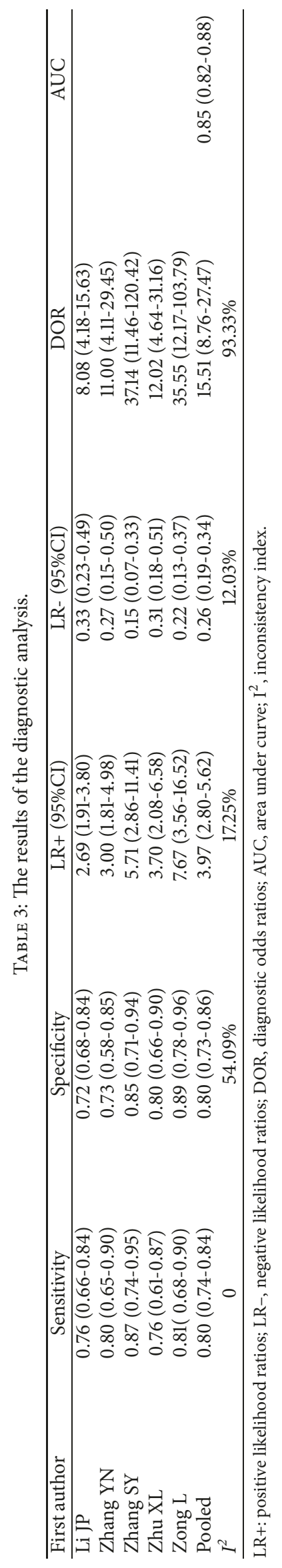




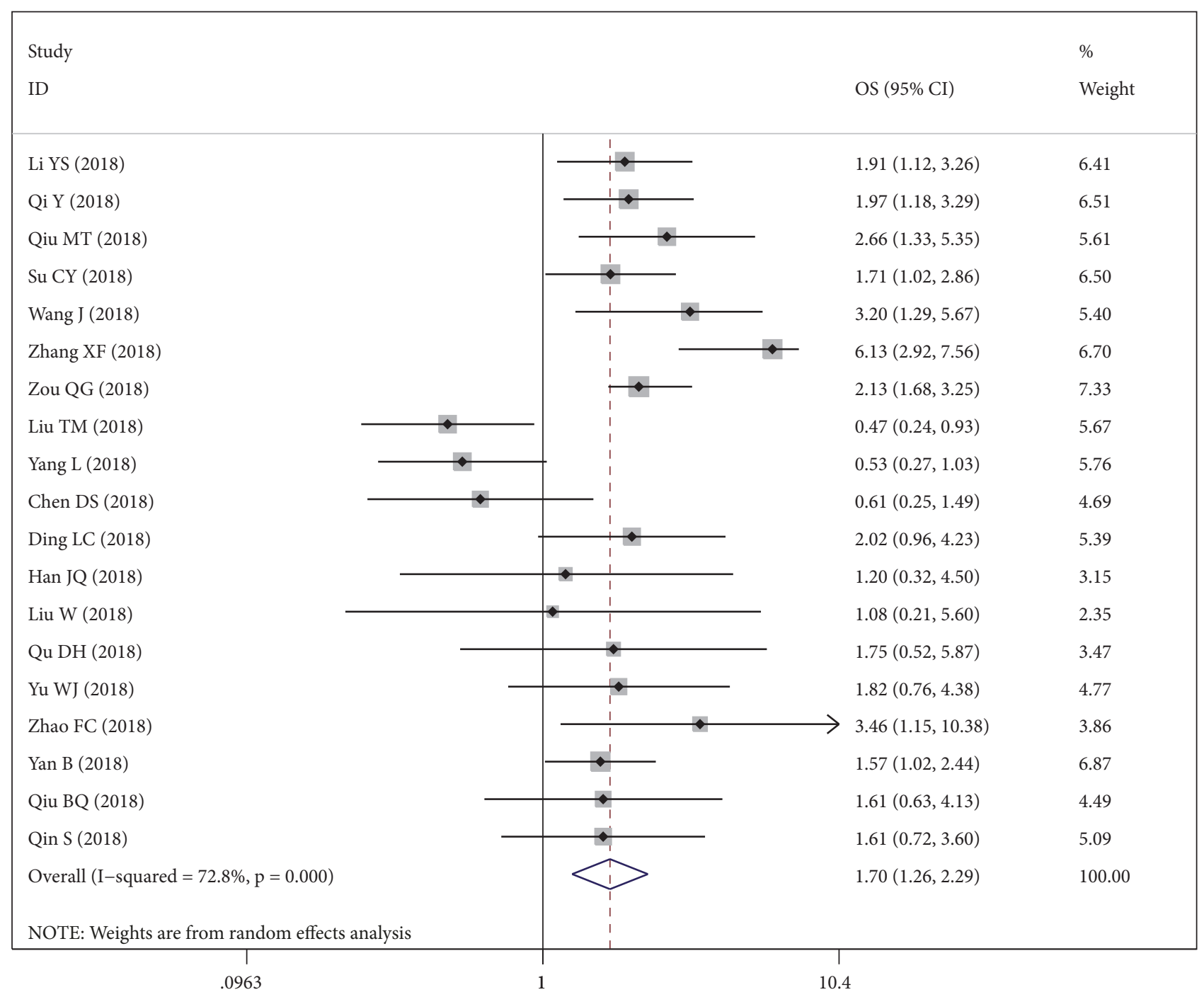

FIGURE 5: Forest plots of the overall prognostic performance of circRNAs.

TABLE 4: The results of the prognostic subgroup analysis.

\begin{tabular}{|c|c|c|c|c|c|c|c|}
\hline Subgroup & No. of studies & HR & LL & $\mathrm{UL}$ & $\mathrm{P}$ & $\mathrm{I}^{2}$ & $\mathrm{P}$ for heterogeneity \\
\hline Total & 1544 & 1.70 & 1.26 & 2.29 & 0.001 & $72.8 \%$ & $<0.001$ \\
\hline Upregulated & 1323 & 2.17 & 1.74 & 2.72 & $<0.001$ & $43.2 \%$ & 0.034 \\
\hline Downregulated & 221 & 0.52 & 0.34 & 0.80 & 0.002 & 0 & $<0.001$ \\
\hline \multicolumn{8}{|l|}{ Analysis methods } \\
\hline Multivariate analysis & 980 & 1.79 & 1.17 & 2.73 & 0.007 & $84.8 \%$ & $<0.001$ \\
\hline Univariate analysis & 564 & 1.56 & 1.13 & 2.16 & 0.007 & 0 & 0.510 \\
\hline \multicolumn{8}{|l|}{ Follow-up time } \\
\hline$\geq 5$ years & 1280 & 1.76 & 1.28 & 2.43 & 0.001 & $72.5 \%$ & $<0.001$ \\
\hline$<5$ years & 258 & 1.52 & 0.67 & 3.47 & 0.319 & $74.9 \%$ & 0.009 \\
\hline
\end{tabular}

HR, hazard ratio; LL, lower limit; UL, upper limit.

articles, results of studies were considered acceptable only based on the expression of circRNAs in tissue, while consequences of studies based on the expression levels of circRNAs in serum, plasma, or peripheral blood samples were not taken into account.
CircFOXO3 was observed with decreased expression in nonsmall cell lung cancer and related to clinical diagnosis with AUC of 0.782 [24]. A study by Lu and his colleagues confirmed that circFOXO3 is significantly downregulated in breast tumor as well [25]. But compared with that in 


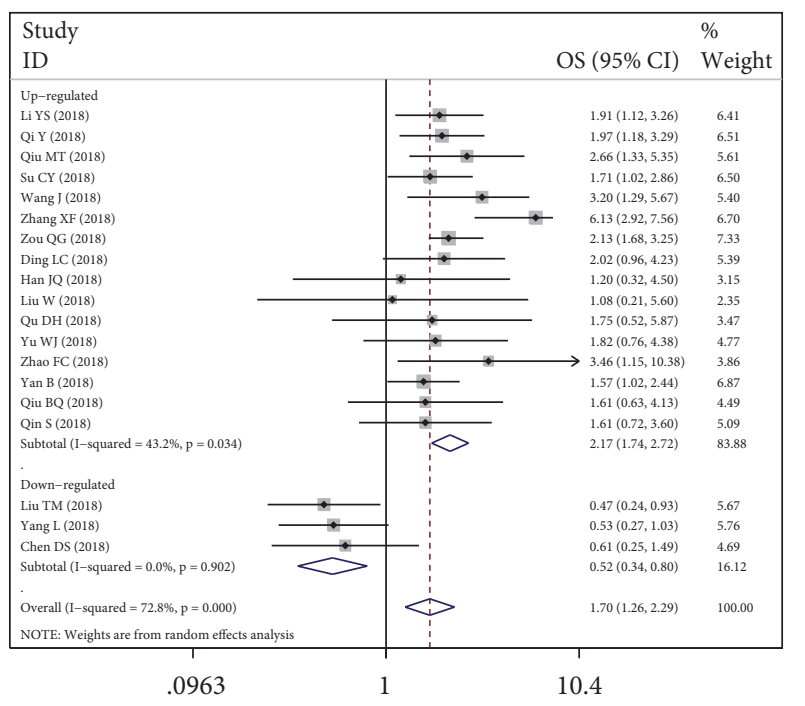

(a)

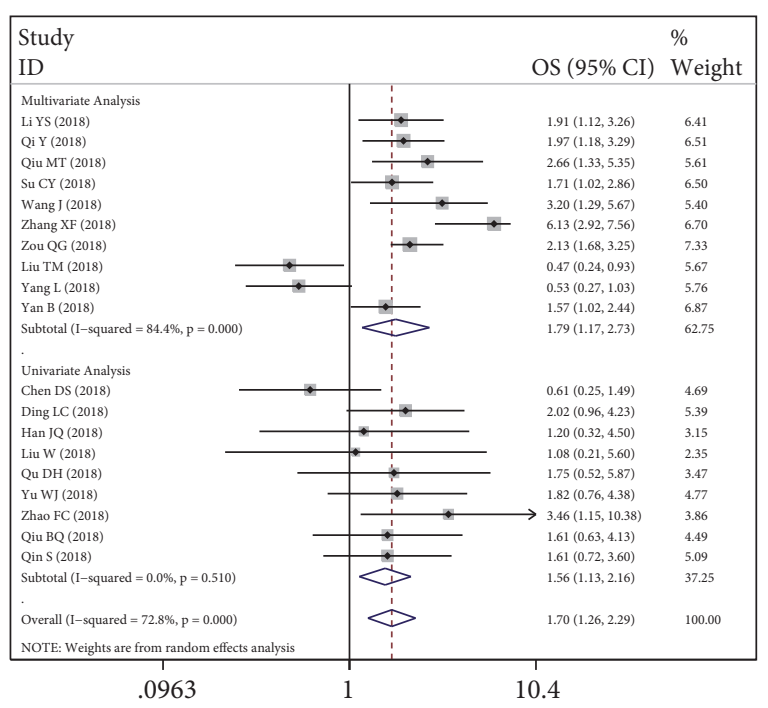

(b)

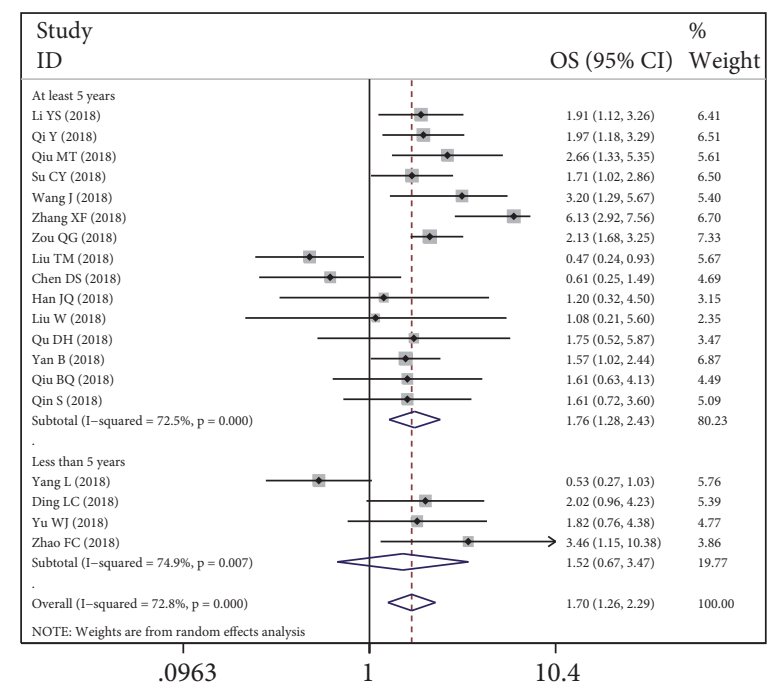

(c)

FIGURE 6: Meta-analysis of subtotal HRs based on (a) upregulated and downregulated circRNAs, (b) analysis methods, and (c) follow-up time.

healthy controls, the expression of hsa_circ_0013958 significantly increased in stage I/II lung adenocarcinoma patients [33]. Similarly, the expression levels of hsa_circ_0079530, hsa_circ_0014130, hsa_circ_102231, and hsa_circ_0000729 were upregulated in lung cancer with good sensitivity and specificity [34-36, 57].

On account of the inconsistent or opposite results of these included studies, several statistical tools were employed to assess the overall diagnostic performance of circRNAs in lung cancer. The sensitivity and specificity were performed to measure the diagnostic value and the pooled sensitivity and specificity were 0.80 (95\%: 0.74-0.84) and 0.80 (95\%: 0.73 $0.86)$, indicating moderate strength to detect lung cancer. In addition, DOR is a single indicator of overall effectiveness of a diagnostic test and when it is greater than one, the test is discriminating correctly [58]. The pooled DOR herein was
15.51 with corresponding 95\% confidence interval from 8.76 to 27.47 and it suggested that circRNAs involved in our study possessed satisfactory ability of diagnosis. Another recommended implement is the AUC of the summary receiver operating characteristic curve (SROC), representing the value of a diagnostic experiment. It is generally recognized that the AUC of SROC with a value more than 0.93 is good and a value ranging from 0.75 to 0.92 is receivable $[59,60]$.

In the current study, the value of AUC was 0.85 (95\%CI: 0.82-0.88). Given the results discussed hereinabove, circRNAs are capable for early detection of lung cancer. Since current conventional serum biomarkers such as carcinoembryonic antigen, cytokeratin 19 fragments 21-1, and neuronspecific enolase are unsatisfactory in both sensitivity and specificity of early detection of lung cancer, circRNAs, with a pooled sensitivity and specificity of 0.8 and 0.8 , respectively, 


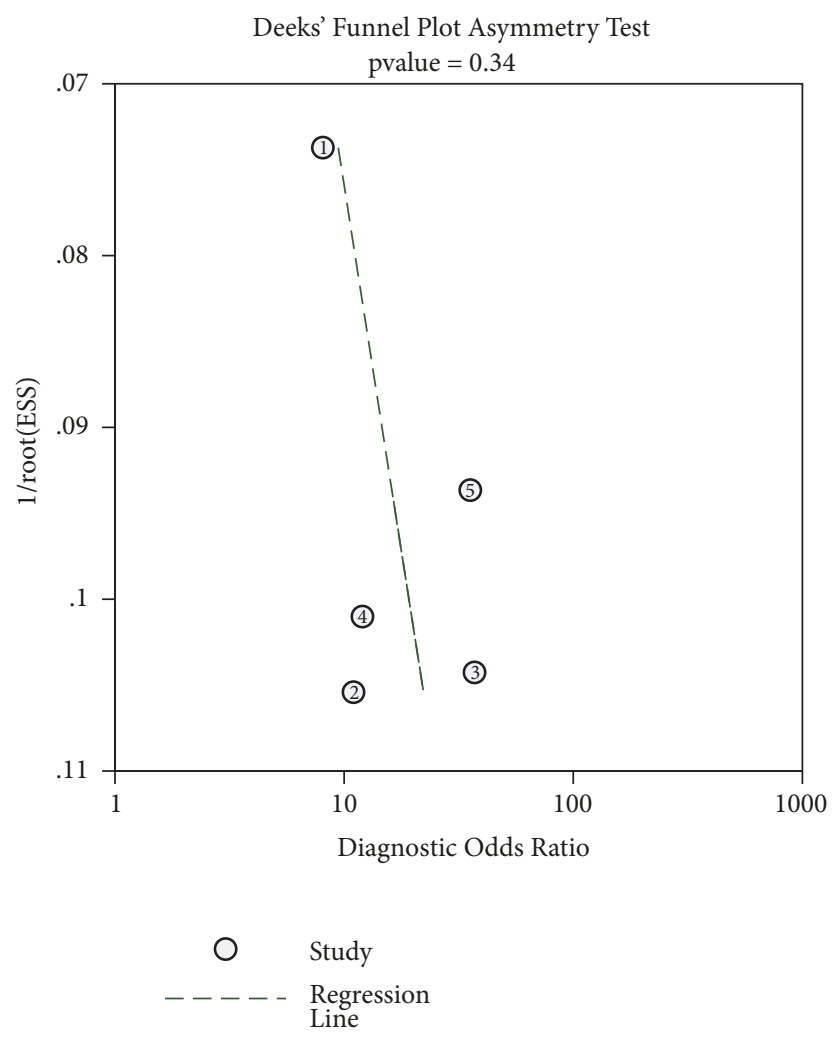

(a)

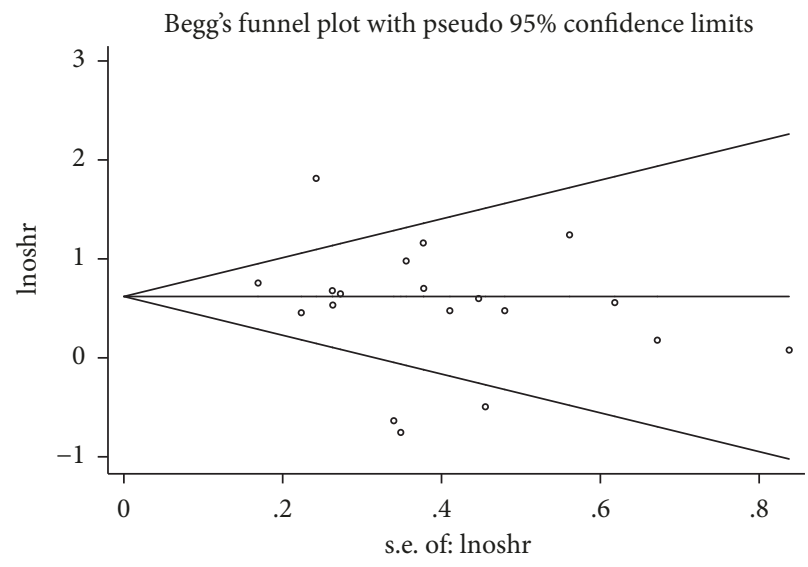

(b)

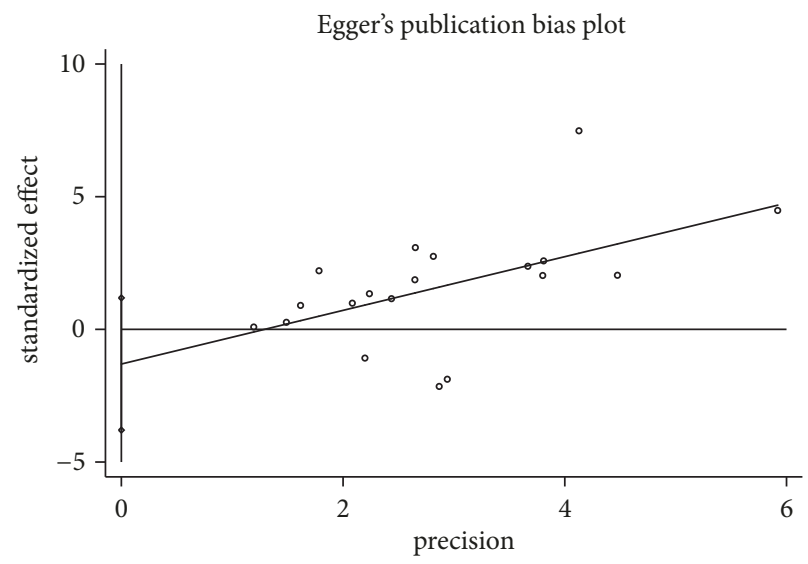

(c)

Figure 7: Funnel plots of publication bias. (a) Deeks' funnel plot asymmetry test for diagnostic studies. (b) Begg's funnel plot for prognostic tests. (c) Egger's funnel plot for prognostic tests.

are relatively hopeful indicators so as to contribute positively to the improvement in the early diagnosis with lung cancer.

Obvious heterogeneity of this diagnostic analysis was assessed; however, we were not able to perform stratified analysis to find out the source of heterogeneity due to lack of sufficient data about some crucial variates concerning design schema, country, ethnicity, age, circRNAs type, controls type, and so on.

4.2. CircRNAs Are Prognostic Biomarkers for Lung Cancer. In the present study, 17 types of circRNAs from 19 studies were identified for prognostic value in lung cancer. All the included studies concentrated on the relationship between aberrant expression of circRNAs and overall survival of lung cancer patients and none of them dealt with other survival indexes like progress free survival. It was mentioned that all patients involved in the study did not receive radiotherapy or chemotherapy before surgery when samples were acquired. Among 19 studies, 3 studies of circRNAs were downregulated, including hsa_circ_0001649, hsa_circ_0046264, and hsa_circ_100395, while the expression levels of the remaining 16 studies of circRNAs were in the opposite. 


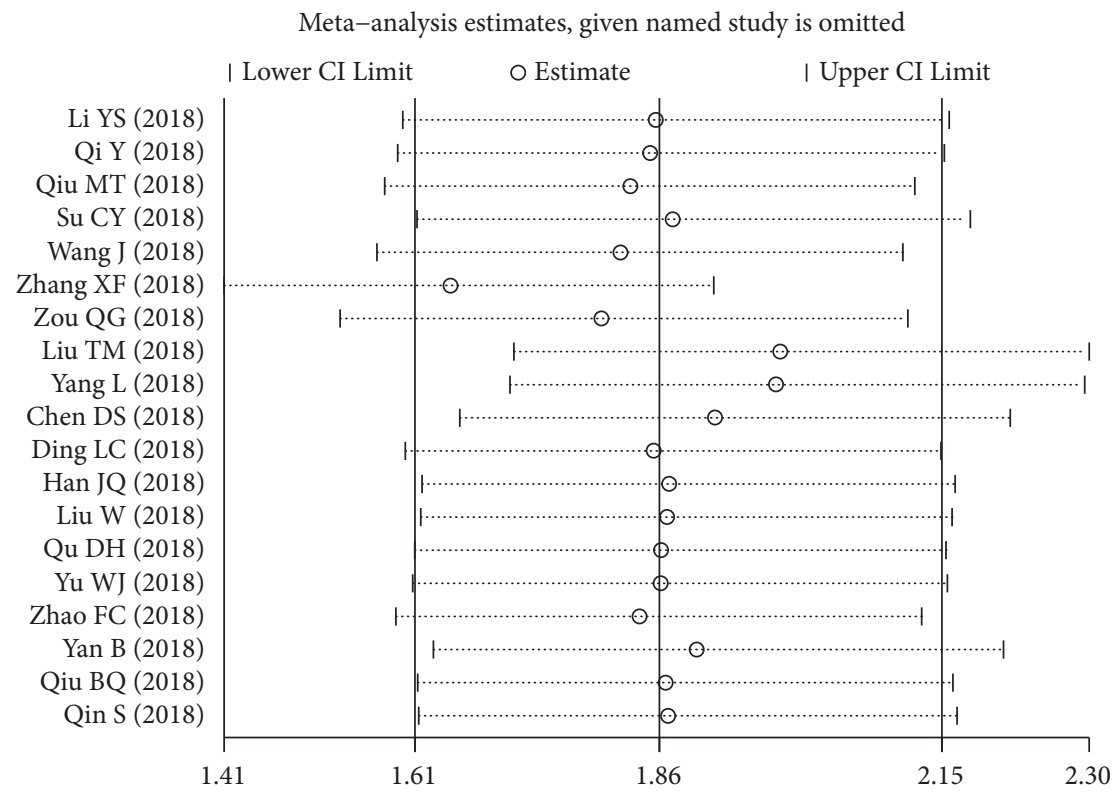

FIGURE 8: Sensitivity analyses for prognostic analysis.

On the whole, our results revealed that the upregulated circRNAs were related to a worse overall survival for lung cancer patients as the pooled HR was 1.70 with 95\% CI from 1.26 to 2.29 . Because there was an evident heterogeneity, subgroup analyses were employed to explore the source. According to the biological function of circRNAs, the upregulated biomarker group showed a lower OS with the pooled HR of $2.17(\mathrm{p}<0.001)$ and pretty low heterogeneity $\left(\mathrm{I}^{2}\right.$ : $43.2 \%$ ), whereas the downregulated group were investigated to have a significantly positive correlation with a stronger prognosis (HR: 0.52, p: 0.002) and improved heterogeneity. Besides, the diversity of analysis methods used in the enrolled research may have an impact on the final results. Multivariate analysis takes into account of all statistical outcome variables at the same time while univariate analysis is conducted with a single factor, considered as the simplest form of quantitative analysis. Generally, multivariate analysis tends to demonstrate higher statistical accuracy than univariate analysis. Similarly, longer follow-up time will be more useful for further evaluation of prognostic values in complex diseases, especially in lung cancer. However, analysis methods in original articles for $\mathrm{HR}$ and corresponding 95\%CI and the length of the time for following up were discovered with nonsignificant association with lung cancer patients' overall survival, meaning that the heterogeneity was not amended after these subset analyses. So we concluded that merging the types of circRNAs with distinctly different biological roles might explain the main source of the heterogeneity.

The overexpression of circPRKCI, circCDRlas, circBANP, and circFADS2 was correlated with unfavorable prognosis in lung cancer. However, circP4HB, circSHPRH, and hsa_circ_100395 were decreased in lung cancer tissues and low expression predicted poor prognosis. Accumulating evidence revealed that the circPRKCI, circCDRlas, circBANP, and circFADS2 functioned as an oncogenic role in lung cancer, whereas the circP4HB, circSHPRH, and hsa_circ_100395 act as tumor suppressor of lung cancer.

In mechanism, aberrant circPRKCI inhibits the cellular proliferation, distant metastasis, and cell invasion in lung cancer by modulating the expression of markers of epithelialto-mesenchymal transition, sponging miR-545 and miR589, and relieving the inhibition of the protumor genic transcription factor E2F7 [44]. Besides, circCDR1as, one of the most frequently studied circRNAs, targets miR-7 in a manner dependent on NF-kB regulatory signaling, upregulates proliferation levels of EGFR, CCNE1, and PIK3CD, and thus induced superior proliferative, migratory, and invasive capabilities of lung cancer cells [12, 32, 49, 61]. Upregulation of ciRS-7 was also identified in colorectal carcinoma and hepatocellular carcinoma with shorter patient survival time than patients with low ciRS-7 expression [30, 31]. What is more, remarkably unregulated circBANP promoted lung cancer cells proliferation and invasion by abrogating the antitumor effects of miR-503/LARP1[39]. Similarly, high expression of circBANP was observed in colorectal carcinoma [62]. Moreover, Circ-FADS2-mediated miR-498 signaling pathway contributes to lung cancer growth and viabilities, and the patients with low expression level of circFADS2 were considered to have favorable clinical outcomes [50]. On the contrary, in basal cell carcinoma and cutaneous squamous cell carcinoma, Sand et al. demonstrated that the two most downregulated circRNAs were derived from the FADS2 gene and they promoted tumor cell proliferation and tumorigenesis $[63,64]$.

In terms of tumor suppressors, circITCH was another well-established molecular biomarker. Expression of circITCH was decreased in colorectal carcinoma [65], lung cancer [66], and esophageal squamous cell carcinoma [67]. CircITCH prevented Wnt/ $\beta$-Catenin pathway from activation and exerted inhibition effects on the progression 
of lung cancer through sponging the miR-7 and miR-214. Hsa_circ_100395 was found to serve as a sponge for TCF 21 in lung cancer and expression level of hsa_circ_100395 was inversely associated with lymph node metastasis and TumorNode-Metastasis stage [37]. CircSHPRH was confirmed as the sponge miR-331-3p and miR-338-5p and thus inhibiting lung cancer cell growth and metastasis [41]. A similar result was observed in Qin's study that the expression of circSHPRH was significantly reduced in hepatocellular carcinoma [17]. As for circP4HB, it promoted apoptosis, yet it arrested cell-cycle progression, restrained proliferation, and reduced cell invasion and migration through upregulating BRCA2 via targeting miR-1245 [47].

Despite the fact that great efforts were paid to fulfill this systematic and comprehensive analysis based on credible quality of included studies, there were still some deficiencies in our study. First, the majority of sample size of subjects is small $[68,69]$. The detection of circRNAs mainly relies on the high throughput sequencing, which is relatively more expensive than traditional detection technology; as a result, the wide clinic application of circRNAs is limited by the costly test method. Equally, the high throughput sequencing, emerging in the last decade, leads to the fact that the researches concentrated on the diagnostic or prognostic roles of circRNA are confined to recent years, most of which are in the year of 2018. Second, the county studied was restricted to China. It was noteworthy that, as a developing country, incidence of various cancer types that occurred in China was different from developed country [70]. Although the morbidity of lung cancer in China accounts for approximately one-third of global new diagnosed cases [1], the imperfection of populations researched narrowed the ranges of applicability in terms of diverse genetic backgrounds and geographic disparity. Third, the heterogeneity of overall diagnostic accuracy and predictive significance was evident, and the potential sources of heterogeneity were not duly clarified by satisfying subgroup analysis owing to insufficient data, which were vital to describe effectiveness of circRNAs in a quantitative manner. Fourth, the samples were extracted merely from lung tissue. CircRNAs that are characterized with closed loop structure, free of poly(A) tails, and the feature which confers them advantageous properties that altered expression of circRNAs are confirmed to be ubiquitous and stable in various human organs and developmental stages. Hence, it was inappropriate to discard records with other sources of samples like peripheral blood mononuclear cell [56] in the literature selection process. Furthermore, given that cancer is something pervasive and stubborn, with sophisticated and underlying mechanisms, it is suggested that combination of several biomarkers might exert better diagnostic accuracy or higher prognostic value of lung cancer than a single biomarker. Besides, more and more tumor markers are implied to appear in a tissue specific manner and serve to distinguish the organogenesis of cancer cells. Thus, further feasible researches are required to spotlight the clinical diagnostic power of detection of multitumor markers from tissue, serum, plasma, and so on, to seek for novel practical methods, to facilitate early diagnosis, and to improve clinical outcomes.

\section{Conclusions}

In summary, this study validates that the altered expressions of circRNAs can be monitored and applied as emerging diagnostic biomarkers with moderate sensitivity and specificity and have satisfactory value in forecasting clinical outcomes of lung cancer patients in China. Nevertheless, well-designed and large-scale researches of multinational clinical trials are further required to verify the results.

$\begin{array}{ll}\text { Abbreviations } \\ \text { AUC: } & \text { Area under the curve } \\ \text { CDRlas: } & \text { Cerebellar degeneration-related protein 1 } \\ & \text { transcript } \\ \text { CI: } & \text { Confidence interval } \\ \text { D: } & \text { Downregulated expression } \\ \text { DOR: } & \text { Diagnostic odds ratio } \\ \text { FN: } & \text { False negative } \\ \text { FP: } & \text { False positive } \\ \text { HR: } & \text { Hazard ratio } \\ \text { LAC: } & \text { Lung adenocarcinoma } \\ \text { NLR: } & \text { Negative likelihood ratio } \\ \text { NSCLC: } & \text { Nonsmall cell lung cancer } \\ \text { U: } & \text { Upregulated expression } \\ \text { OS: } & \text { Overall survival } \\ \text { PLR: } & \text { Positive likelihood ratio } \\ \text { QUADAS: } & \text { Quality Assessment of Diagnostic } \\ & \text { Accuracy Studies } \\ \text { qRT-PCR: } & \text { Real-time polymerase chain reaction } \\ \text { SROC: } & \text { Summary receiver operating curve } \\ \text { TN: } & \text { True negative } \\ \text { TP: } & \text { True positive. }\end{array}$

\section{Data Availability}

The data used to support the findings of this study are included within the article.

\section{Conflicts of Interest}

The authors have declared that no conflicts of interest exist.

\section{Authors' Contributions}

Chengdi Wang, Yuting Jiang, and Qian Lei are equal contributors and co-first authors.

\section{Acknowledgments}

This work was supported by the Science and Technology Project of Chengdu (2017-CY02-00030-GX) and Special Funds for Local Science and Technology Development Guided by the Central Government (2016CZYD0001).

\section{Supplementary Materials}

Figure S1: quality evaluation of diagnostic accuracy for the enrolled studies as well as risk of bias and applicability 
concerns' (A) graph and (B) summary. (Supplementary Materials)

\section{References}

[1] F. Bray, J. Ferlay, I. Soerjomataram, R. L. Siegel, L. A. Torre, and A. Jemal, "Global cancer statistics 2018: GLOBOCAN estimates of incidence and mortality worldwide for 36 cancers in 185 countries," CA: A Cancer Journal for Clinicians, vol. 68, no. 6, pp. 394-424, 2018.

[2] Y. Cheng, M. P. A. Davies, D. Liu, W. Li, and J. K. Field, "Implementation planning for lung cancer screening in China," Precision Clinical Medicine, vol. 2, no. 1, pp. 13-14, 2019.

[3] S. Peters, A. A. Adjei, C. Gridelli, M. Reck, K. Kerr, and E. Felip, "Metastatic non-small-cell lung cancer (NSCLC): ESMO Clinical Practice Guidelines for diagnosis, treatment and follow-up," Annals of Oncology, vol. 23, no. suppl 7, pp. vii56vii64, 2012.

[4] R. S. Herbst, D. Morgensztern, and C. Boshoff, "The biology and management of non-small cell lung cancer," Nature, vol. 553, no. 7689, pp. 446-454, 2018.

[5] Y. Li, Q. Zheng, C. Bao et al., "Circular RNA is enriched and stable in exosomes: a promising biomarker for cancer diagnosis," Cell Research, vol. 25, no. 8, pp. 981-984, 2015.

[6] W. R. Jeck and N. E. Sharpless, "Detecting and characterizing circular RNAs," Nature Biotechnology, vol. 32, no. 5, pp. 453-461, 2014.

[7] J. N. Vo, M. Cieslik, Y. Zhang et al., "The landscape of circular RNA in cancer," Cell, vol. 176, no. 4, pp. 869-881, 2019.

[8] H. L. Sanger, G. Klotz, D. Riesner, H. J. Gross, and A. K. Kleinschmidt, "Viroids are single stranded covalently closed circular RNA molecules existing as highly base paired rod like structures," Proceedings of the National Acadamy of Sciences of the United States of America, vol. 73, no. 11, pp. 3852-3856, 1976.

[9] J. U. Guo, V. Agarwal, H. Guo, and D. P. Bartel, "Expanded identification and characterization of mammalian circular RNAs," Genome Biology, vol. 15, no. 7, article no. 409, 2014.

[10] Z. Li, C. Huang, C. Bao et al., "Exon-intron circular RNAs regulate transcription in the nucleus," Nature Structural \& Molecular Biology, vol. 22, no. 3, pp. 256-264, 2015.

[11] J. Salzman, R. E. Chen, M. N. Olsen, P. L. Wang, and P. O. Brown, "Cell-type specific features of circular RNA expression," PLoS Genetics, vol. 9, no. 9, Article ID e1003777, 2013.

[12] S. Memczak, M. Jens, A. Elefsinioti et al., "Circular RNAs are a large class of animal RNAs with regulatory potency," Nature, vol. 495, no. 7441, pp. 333-338, 2013.

[13] S. J. Conn, K. A. Pillman, J. Toubia et al., "The RNA binding protein quaking regulates formation of circRNAs," Cell, vol. 160, no. 6, pp. 1125-1134, 2015.

[14] W. R. Jeck, J. A. Sorrentino, K. Wang et al., "Circular RNAs are abundant, conserved, and associated with ALU repeats," RNA, vol. 19, no. 2, pp. 141-157, 2013.

[15] P. Li, S. Chen, H. Chen et al., "Using circular RNA as a novel type of biomarker in the screening of gastric cancer," Clinica Chimica Acta, vol. 444, pp. 132-136, 2015.

[16] X. Wang, Y. Zhang, L. Huang et al., "Decreased expression of hsa_circ_001988 in colorectal cancer and its clinical significances," International Journal of Clinical and Experimental Pathology, vol. 8, no. 12, pp. 16020-16025, 2015.

[17] M. Qin, G. Liu, X. Huo et al., "Hsa_circ_0001649: a circular RNA and potential novel biomarker for hepatocellular carcinoma," Cancer Biomarkers, vol. 16, no. 1, pp. 161-169, 2016.
[18] X. Shang, G. Li, H. Liu et al., "Comprehensive circular RNA profiling reveals that hsa-circ-0005075, a new circular RNA biomarker, is involved in hepatocellular crcinoma development," Medicine, vol. 95, no. 22, Article ID e3811, 2016.

[19] X. Song, N. Zhang, P. Han et al., "Circular RNA profile in gliomas revealed by identification tool UROBORUS," Nucleic Acids Research, vol. 44, no. 9, article no. e87, 2016.

[20] W. Xia, M. Qiu, R. Chen et al., "Circular RNA has-circ-0067934 is upregulated in esophageal squamous cell carcinoma and promoted proliferation," Scientific Reports, vol. 6, p. 35576, 2016.

[21] L. Xuan, L. Qu, H. Zhou et al., "Circular RNA: a novel biomarker for progressive laryngeal cancer," American Journal of Translational Research, vol. 8, no. 2, pp. 932-939, 2016.

[22] X. Cui, J. Wang, Z. Guo et al., "Emerging function and potential diagnostic value of circular RNAs in cancer," Molecular Cancer, vol. 17, no. 1, p. 123, 2018.

[23] J. Zhao, L. Li, Q. Wang, H. Han, Q. Zhan, and M. Xu, "CircRNA expression profile in early-stage lung adenocarcinoma patients," Cellular Physiology and Biochemistry, vol. 44, no. 6, pp. 21382146, 2018.

[24] Y. Zhang, H. Zhao, and L. Zhang, "Identification of the tumorsuppressive function of circular RNA FOXO3 in non-small cell lung cancer through sponging miR-155," Molecular Medicine Reports, vol. 17, no. 6, pp. 7692-7700, 2018.

[25] W. Lu, "Roles of the circular RNA circ-Foxo3 in breast cancer progression," Cell Cycle, vol. 16, no. 7, pp. 589-590, 2017.

[26] T. B. Hansen, J. Kjems, and C. K. Damgaard, "Circular RNA and miR-7 in cancer,” Cancer Research, vol. 73, no. 18, pp. 5609-5612, 2013.

[27] A. M. Cheng, M. W. Byrom, J. Shelton, and L. P. Ford, "Antisense inhibition of human miRNAs and indications for an involvement of miRNA in cell growth and apoptosis," Nucleic Acids Research, vol. 33, no. 4, pp. 1290-1297, 2005.

[28] Y.-T. Chou, H.-H. Lin, Y.-C. Lien et al., "EGFR promotes lung tumorigenesis by activating miR-7 through a Ras/ERK/Myc pathway that targets the Ets2 transcriptional repressor ERF," Cancer Research, vol. 70, no. 21, pp. 8822-8831, 2010.

[29] A. Bachmayr-Heyda, A. T. Reiner, K. Auer et al., "Correlation of circular RNA abundance with proliferation-exemplified with colorectal and ovarian cancer, idiopathic lung fibrosis, and normal human tissues," Scientific Reports, vol. 5, article 8057, 2015.

[30] W. Weng, Q. Wei, S. Toden et al., "Circular RNA ciRS-7-a promising prognostic biomarker and a potential therapeutic target in colorectal cancer," Clinical Cancer Research, vol. 23, no. 14, pp. 3918-3928, 2017.

[31] L. Xu, M. Zhang, X. Zheng, P. Yi, C. Lan, and M. Xu, "The circular RNA ciRS-7 (Cdrlas) acts as a risk factor of hepatic microvascular invasion in hepatocellular carcinoma," Journal of Cancer Research and Clinical Oncology, vol. 143, no. 1, pp. 17-27, 2017.

[32] C. Su, Y. Han, H. Zhang et al., "CiRS-7 targeting miR-7 modulates the progression of non-small cell lung cancer in a manner dependent on NF- $\kappa$ B signalling," Journal of Cellular and Molecular Medicine, vol. 22, no. 6, pp. 3097-3107, 2018.

[33] X. Zhu, X. Wang, S. Wei et al., "hsa_circ_0013958: a circular RNA and potential novel biomarker for lung adenocarcinoma," FEBS Journal, vol. 284, no. 14, pp. 2170-2182, 2017.

[34] J. Li, J. Wang, Z. Chen, Y. Chen, and M. Jin, "Hsa_circ_0079530 promotes cell proliferation and invasion in non-small cell lung cancer," Gene, vol. 665, pp. 1-5, 2018. 
[35] S. Zhang, X. Zeng, T. Ding et al., "Microarray profile of circular RNAs identifies hsa-circ-0014130 as a new circular RNA biomarker in non-small cell lung cancer," Scientific Reports, vol. 8, no. 1, p. 2878, 2018.

[36] L. Zong, Q. Sun, H. Zhang et al., "Increased expression of circRNA_102231 in lung cancer and its clinical significance," Biomedicine \& Pharmacotherapy, vol. 102, pp. 639-644, 2018.

[37] D. Chen, W. Ma, Z. Ke, and F. Xie, "CircRNA hsa_circ_100395 regulates miR-1228/TCF21 pathway to inhibit lung cancer progression," Cell Cycle, vol. 17, no. 16, pp. 2080-2090, 2018.

[38] L. Ding, W. Yao, J. Lu, J. U. N. Gong, and X. Zhang, "Upregulation of circ_001569 predicts poor prognosis and promotes cell proliferation in non-small cell lung cancer by regulating the Wnt/ $\beta$-catenin pathway," Oncology Letters, vol. 16, no. 1, pp. 453-458, 2018.

[39] J. Han, G. Zhao, X. Ma et al., "CircRNA circ-BANP-mediated miR-503/LARP1 signaling contributes to lung cancer progression," Biochemical and Biophysical Research Communications, vol. 503, no. 4, pp. 2429-2435, 2018.

[40] Y. Li, J. Hu, L. Li et al., "Upregulated circular RNA circ_0016760 indicates unfavorable prognosis in NSCLC and promotes cell progression through miR-1287/GAGE1 axis," Biochemical and Biophysical Research Communications, vol. 503, no. 3, pp. 20892094, 2018.

[41] T. Liu, Z. Song, and Y. Gai, "Circular RNA circ_0001649 acts as a prognostic biomarker and inhibits NSCLC progression via sponging miR-331-3p and miR-338-5p," Biochemical and Biophysical Research Communications, vol. 503, no. 3, pp. 15031509, 2018.

[42] W. Liu, W. Ma, Y. Yuan, Y. Zhang, and S. Sun, "Circular RNA hsa_circRNA_103809 promotes lung cancer progression via facilitating ZNF121-dependent MYC expression by sequestering miR-4302," Biochemical and Biophysical Research Communications, vol. 500, no. 4, pp. 846-851, 2018.

[43] Y. Qi, B. Zhang, J. Wang, and M. Yao, "Upregulation of circular RNA hsa_circ_0007534 predicts unfavorable prognosis for NSCLC and exerts oncogenic properties in vitro and in vivo," Gene, vol. 676, pp. 79-85, 2018.

[44] M. Qiu, W. Xia, R. Chen et al., "The circular RNA circPRKCI promotes tumor growth in lung adenocarcinoma," Cancer Research, vol. 78, no. 11, pp. 2839-2851, 2018.

[45] D. Qu, B. Yan, R. Xin, and T. Ma, "A novel circular RNA hsa_circ_0020123 exerts oncogenic properties through suppression of miR-144 in non-small cell lung cancer," American Journal of Cancer Research, vol. 8, no. 8, pp. 1387-1402, 2018.

[46] J. Wang and H. Li, "CircRNA circ-0067934 silencing inhibits the proliferation, migration and invasion of NSCLC cells and correlates with unfavorable prognosis in NSCLC," European Review for Medical and Pharmacological Sciences, vol. 22, no. 10, pp. 3053-3060, 2018.

[47] L. Yang, J. Wang, Y. Fan, K. Yu, B. Jiao, and X. Su, "Hsa_circ_0046264 up-regulated BRCA2 to suppress lung cancer through targeting hsa-miR-1245," Respiratory Research, vol. 19, no. 1, p. 115, 2018.

[48] W. Yu, H. Jiang, H. Zhang, and J. Li, "Hsa_circ_0003998 promotes cell proliferation and invasion by targeting miR-326 in non-small cell lung cancer," OncoTargets and Therapy, vol. 11, pp. 5569-5577, 2018.

[49] X. Zhang, D. Yang, and Y. Wei, "Overexpressed CDRlas functions as an oncogene to promote the tumor progression via miR7 in non-small-cell lung cancer," OncoTargets and Therapy, vol. 11, pp. 3979-3987, 2018.
[50] F. Zhao, Y. Han, Z. Liu, Z. Zhao, Z. Li, and K. Jia, "CircFADS2 regulates lung cancer cells proliferation and invasion via acting as a sponge of miR-498," Bioscience Reports, vol. 38, no. 4, Article ID BSR20180570, 2018.

[51] Q. Zou, T. Wang, B. Li et al., "Overexpression of circ-0067934 is associated with increased cellular proliferation and the prognosis of non-small cell lung cancer," Oncology Letters, vol. 16, no. 5, pp. 5551-5556, 2018.

[52] B.-Q. Qiu, P.-F. Zhang, D. Xiong et al., "CircRNA fibroblast growth factor receptor 3 promotes tumor progression in nonsmall cell lung cancer by regulating Galectin-1-AKT/ERK1/2 signaling," Journal of Cellular Physiology, vol. 234, no. 7, pp. 11256-11264, 2018.

[53] S. Qin, Y. Zhao, G. Lim, H. Lin, and X. Zhang, "Circular RNA PVT1 acts as a competing endogenous RNA for miR-497 in promoting non-small cell lung cancer progression," Biomedicine \& Pharmacotherapy, vol. 111, pp. 244-250, 2018.

[54] B. Yan, W. Zhang, X.-W. Mao, and L.-Y. Jiang, "Circular RNA ciRS-7 correlates with advance disease and poor prognosis, and its down-regulation inhibits cells proliferation while induces cells apoptosis in non-small cell lung cancer," European Review for Medical and Pharmacological Sciences, vol. 22, no. 24, pp. 8712-8721, 2018.

[55] S. Tan, Q. Gou, W. Pu et al., "Circular RNA F-circEA produced from EML4-ALK fusion gene as a novel liquid biopsy biomarker for non-small cell lung cancer," Cell Research, vol. 28, no. 6, pp. 693-695, 2018.

[56] F. Wang, A. J. Nazarali, and S. Ji, "Circular RNAs as potential biomarkers for cancer diagnosis and therapy," American Journal of Cancer Research, vol. 6, no. 6, pp. 1167-1176, 2016.

[57] S. Li, X. Sun, S. Miao et al., "hsa_circ_0000729, a potential prognostic biomarker in lung adenocarcinoma," Thoracic Cancer, vol. 9, no. 8, pp. 924-930, 2018.

[58] A. S. Glas, J. G. Lijmer, M. H. Prins, G. J. Bonsel, and P. M. M. Bossuyt, "The diagnostic odds ratio: a single indicator of test performance," Journal of Clinical Epidemiology, vol. 56, no. 11, pp. 1129-1135, 2003.

[59] S. D. Walter, "Properties of the summary receiver operating characteristic (SROC) curve for diagnostic test data," Statistics in Medicine, vol. 21, no. 9, pp. 1237-1256, 2002.

[60] C. M. Jones and T. Athanasiou, "Summary receiver operating characteristic curve analysis techniques in the evaluation of diagnostic tests," The Annals of Thoracic Surgery, vol. 79, no. 1, pp. 16-20, 2005.

[61] T. B. Hansen, T. I. Jensen, B. H. Clausen et al., "Natural RNA circles function as efficient microRNA sponges," Nature, vol. 495, no. 7441, pp. 384-388, 2013.

[62] M. Zhu, Y. Xu, Y. Chen, and F. Yan, "Circular BANP, an upregulated circular RNA that modulates cell proliferation in colorectal cancer," Biomedicine \& Pharmacotherapy, vol. 88, pp. 138-144, 2017.

[63] M. Sand, F. G. Bechara, T. Gambichler et al., "Circular RNA expression in cutaneous squamous cell carcinoma," Journal of Dermatological Science, vol. 83, no. 3, pp. 210-218, 2016.

[64] M. Sand, F. G. Bechara, D. Sand et al., "Circular RNA expression in basal cell carcinoma," Epigenomics, vol. 8, no. 5, pp. 619-632, 2016.

[65] G. Huang, H. Zhu, Y. Shi, W. Wu, H. Cai, and X. Chen, "CirITCH plays an inhibitory role in colorectal cancer by regulating the wnt/ $\beta$-catenin pathway," PLoS ONE, vol. 10 , no. 6 , article e0131225, 2015. 
[66] L. Wan, L. Zhang, K. Fan, Z.-X. Cheng, Q.-C. Sun, and J.J. Wang, "Circular RNA-ITCH suppresses lung cancer proliferation via inhibiting the Wnt/ $\beta$-catenin pathway," BioMed Research International, vol. 2016, Article ID 1579490, 2016.

[67] F. Li, L. Zhang, W. Li et al., "Circular RNA ITCH has inhibitory effect on ESCC by suppressing the Wnt/ $\beta$-catenin pathway," Oncotarget, vol. 6, no. 8, pp. 6001-6013, 2015.

[68] J. P. A. Ioannidis, "Why most discovered true associations are inflated," Epidemiology, vol. 19, no. 5, pp. 640-648, 2008.

[69] G. Rucker, G. Schwarzer, J. R. Carpenter, H. Binder, and M. Schumacher, "Treatment-effect estimates adjusted for smallstudy effects via a limit meta-analysis," Biostatistics, vol. 12, no. 1, pp. 122-142, 2010.

[70] W. Chen, R. Zheng, P. D. Baade et al., "Cancer statistics in China, 2015," CA: A Cancer Journal for Clinicians, vol. 66, no. 2, pp. 115132, 2016. 


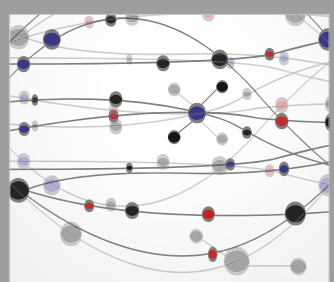

The Scientific World Journal
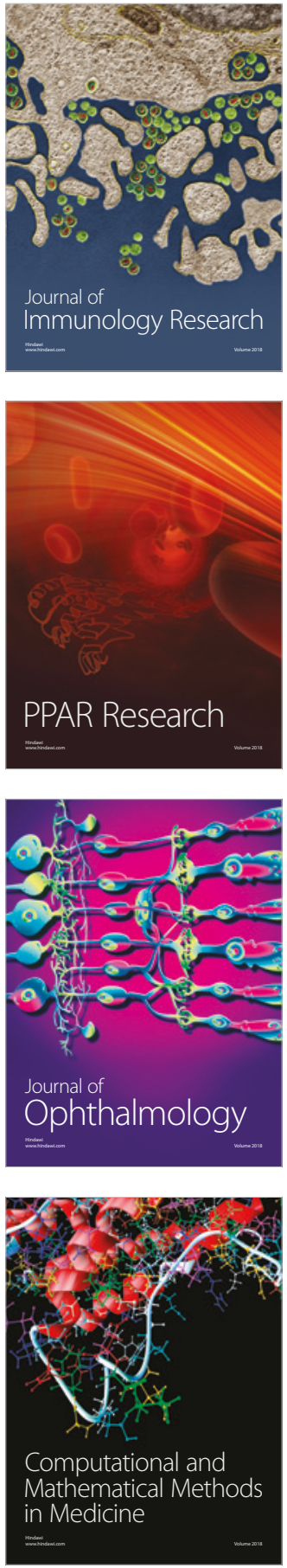

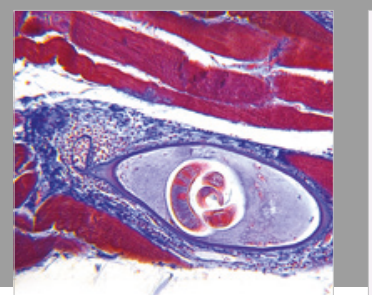

Gastroenterology Research and Practice

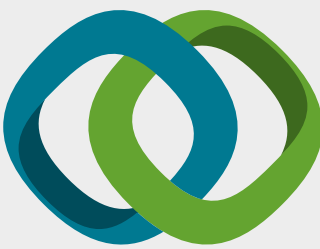

\section{Hindawi}

Submit your manuscripts at

www.hindawi.com
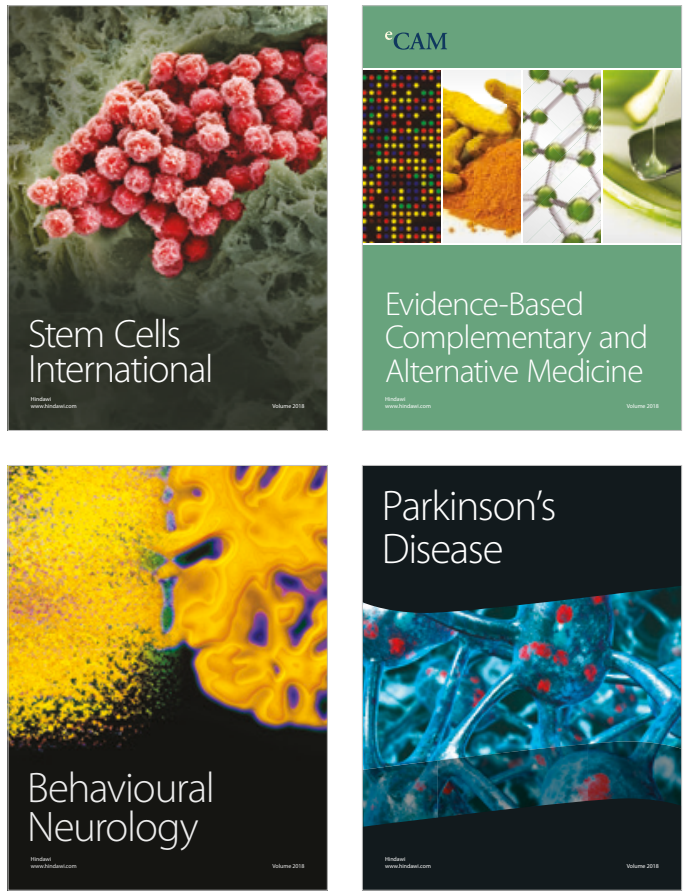

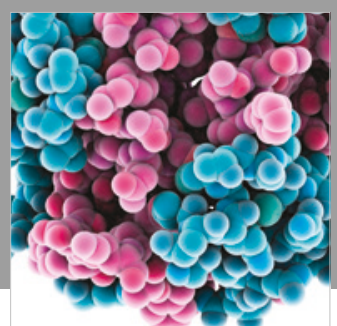

ournal of

Diabetes Research

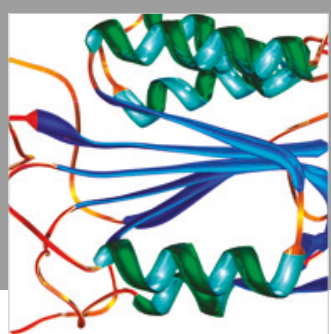

Disease Markers
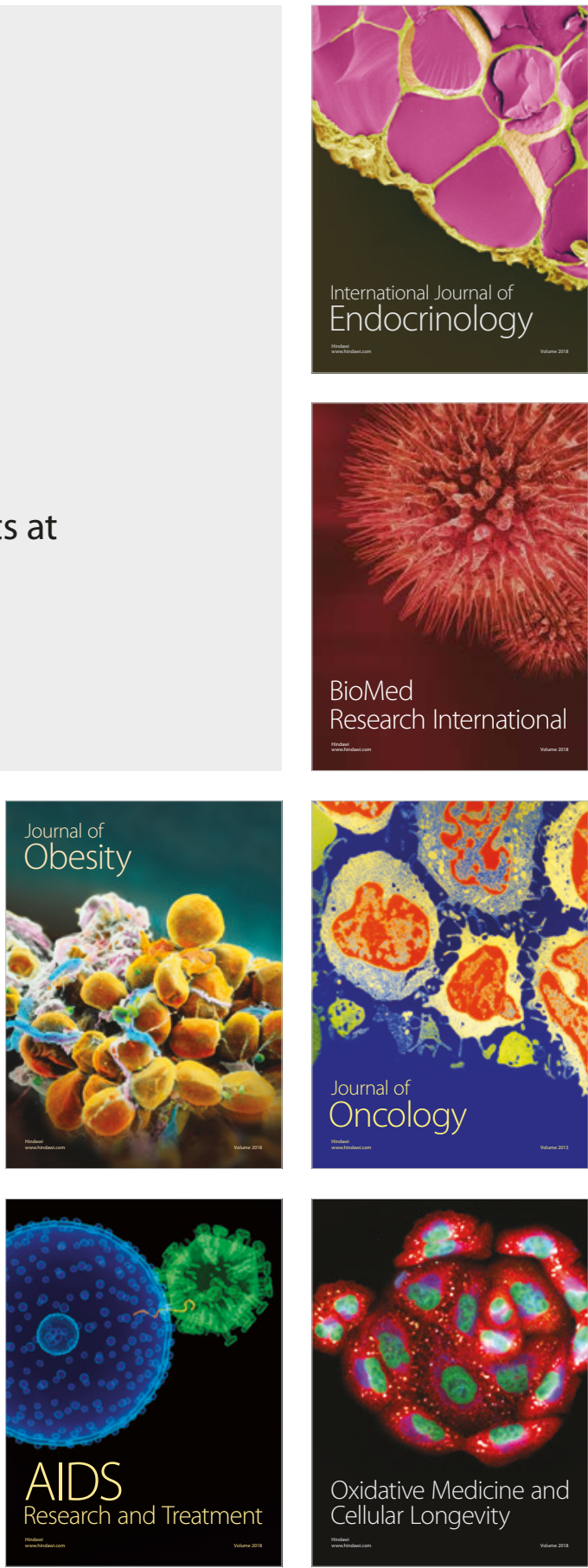